\title{
Low-Reynolds Number Wing Response to an Oscillating Freestream with and without Feed Forward Control
}

\author{
David Williams $^{1}$, Vien Quach ${ }^{2}$, Wesley Kerstens ${ }^{2}$, Seth Buntain ${ }^{2}$ \\ Illinois Institute of Technology, Chicago, IL, 60616 \\ Gilead Tadmor ${ }^{3}$ \\ Northeastern University, Boston, MA, 02115, \\ Clarence Rowley ${ }^{4}$ \\ Princeton University, Princeton, NJ, 08544, \\ and \\ Tim Colonius ${ }^{5}$ \\ California Institute of Technology, Pasadena, CA, 91125
}

\begin{abstract}
The unsteady lift of a low Reynolds number wing in an oscillating freestream is documented in terms of its amplitude and phase. The phase variation of the lift relative to the freestream velocity shows a larger phase difference than predicted by classical unsteady flow theory. A constant time delay between the lift and the actuator was observed to be $\tau^{+}=t_{\text {delay }} \mathbf{U} / \mathbf{c}=5.3$ when normalized by the freestream speed and chord. Feed forward control of pulsed-jet actuators is used to modulate the lift coefficient of the wing, in an attempt to suppress the lift oscillations. Suppression of the fluctuating lift at the fundamental frequency was partially successful, but additional "noise" was added to harmonics of the lift signal by the controller.
\end{abstract}

\section{Nomenclature}

$A R=$ aspect ratio, $(2 \mathrm{c})^{2} / \mathrm{S}$

$C_{L} \quad=$ lift coefficient of wing

$\mathrm{C}_{\mathrm{L}, \alpha}=$ lift curve slope, $\mathrm{dC}_{\mathrm{L}} / \mathrm{d} \alpha$

c $\quad=$ chord

$\mathrm{f}^{+} \quad=$ normalized frequency, $\mathrm{fc} / \mathrm{U}$

$\mathrm{k}=$ normalized frequency, $\pi \mathrm{fc} / \mathrm{U}$

$\mathrm{q}=$ dynamic pressure, $\frac{1}{2} \rho U^{2}$

$S \quad=$ planform area

$U^{\prime}=$ longitudinal velocity increment

$\mathrm{U}_{\mathrm{o}} \quad=$ wing flight speed

$\alpha=$ angle of attack

$\tau^{+} \quad=$ normalized time delay, $\mathrm{t}_{\text {delay }} \mathrm{U} / \mathrm{c}$

\footnotetext{
${ }^{1}$ Professor, Mechanical and Aerospace Engineering Department, and AIAA Associate Fellow.

${ }^{2}$ Undergraduate Research Assistant, Mechanical and Aerospace Engineering Department.

${ }^{3}$ Professor, Electrical and Computer Engineering Department, and AIAA Member.

${ }^{4}$ Associate Professor, Mechanical and Aerospace Engineering Dept., and AIAA Member.

${ }^{5}$ Professor, Mechanical Engineering Department, and AIAA Senior Member.
} 


\section{Introduction}

STABILIZED flight through unsteady and gusting flows must take into account both longitudinal and vertical components of velocity disturbances. Conventional gust analysis described by Ref. 1 shows that vertical velocity fluctuations change the instantaneous angle of attack of the wing, while longitudinal velocity fluctuations affect lift through changes in the dynamic pressure. The incremental load factors (lift/weight of aircraft) acting on the flight vehicle in unsteady flows is proportional to the flight speed for vertical disturbances, but the load factor associated with longitudinal disturbances is inversely proportional to flight speed. For normal sized aircraft the vertical disturbances produce the largest load factors, but the load factors associated with longitudinal disturbances become comparable at low flight speeds and high lift coefficients typical of micro air vehicles.

Active flow control has been used by many investigators to increase the lift coefficient on partially stalled airfoils in steady flight conditions. In this study we examine the feasibility of using active flow control to modulate the lift coefficient in response to changing flight conditions. This type of "dynamic" active flow control introduces a new time delay into the problem of gust suppression, which is the finite response time for the wing's lift to adjust to the actuator input. Another important time delay is the lift response relative to the changing freestream condition. To achieve effective gust suppression it is necessary to accurately account for both time delays.

The effects of an unsteady longitudinal flow on the lift produced by a low aspect ratio wing are measured using a wind tunnel capable of producing sinusoidal speed oscillations. The oscillating freestream simulates the longitudinal component of a gust. The wing's time varying lift is recorded to measure the time delay between the lift and freestream flow. The response of the wing to the actuator input is acquired under steady flow conditions to measure the actuator time delay. The two time delays are then combined to form a feed forward control algorithm based on a quasi-steady aerodynamics model. The controller adjusts the lift coefficient in such a way that the oscillatory component of lift is

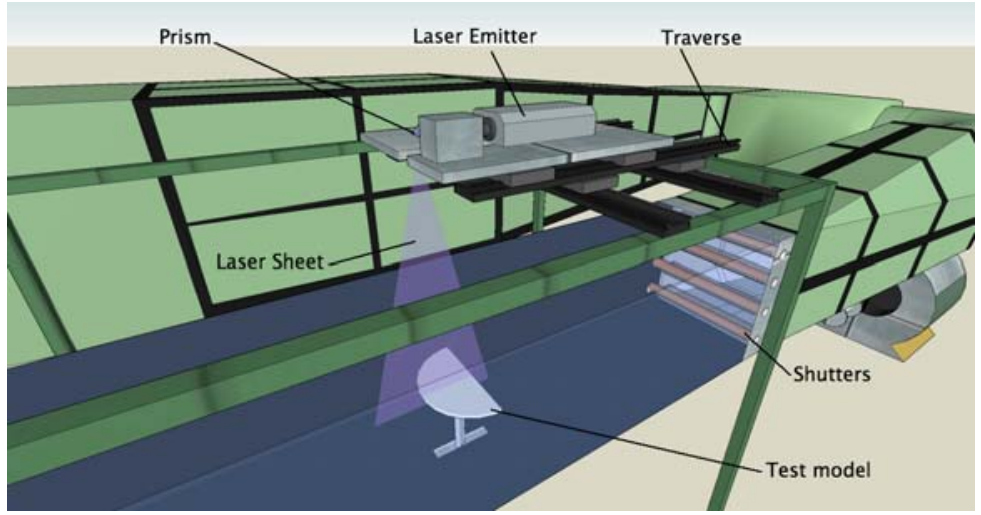

Figure 1. Andrew Fejer Unsteady Flow Wind Tunnel. Semicircular wing is shown in center of test section. Shutter mechanism at the downstream end of the test section produces the flow oscillations.

offset, in an attempt to suppress lift fluctuations. The original plan was to first perform the experiments under quasi-steady conditions, and then slowly increment the frequency of oscillation until the unsteady effects became important. However, as discussed below, the unsteady effects became important much sooner than anticipated.

\section{Experimental Setup}

The Andrew Fejer Unsteady Flow Wind Tunnel is used for the measurements discussed in this paper. The test section has a $610 \mathrm{~mm} \times 610 \mathrm{~mm}$ cross section. The freestream speed is controlled by a shutter system located at the downstream end of the test section as shown in Figure 1. The mean flow speed is $2.85 \mathrm{~m} / \mathrm{s}$ or $5.25 \mathrm{~m} / \mathrm{s}$ depending on the specific experiment, giving chord based Reynolds numbers ranging from 40,000 to 71,000. The corresponding freestream turbulence at a mean speed of $3 \mathrm{~m} / \mathrm{s}$ was measured to be 0.6 percent over a bandwidth of $0.1 \mathrm{~Hz}-30 \mathrm{~Hz}$. The freestream oscillation amplitude settings are varied from 3 percent to 10 percent of the mean speed. The shutter motion is controlled by a PC computer and printed-circuit motor ( $2 \mathrm{~kW}$ PMI), which produces frequencies up to $3 \mathrm{~Hz}$ sinusoidal oscillation in the streamwise velocity component. The distortion in the freestream oscillation is quantified by measuring the first harmonic amplitude, which is less than $20 \mathrm{~dB}$ of the fundamental.

The wing has a semi-circular planform with a span of $406 \mathrm{~mm}$ and a centerline chord of $203 \mathrm{~mm}$. The aspect ratio is 2.54. The curved portion of the wing is aligned as the leading edge, which gives a continuously changing sweep angle from $0^{\circ}$ on centerline to $90^{\circ}$ at the wing tips. The wing model is constructed from Duraform ${ }^{\mathrm{tm}}$ nylon using a Selective Laser Sintering rapid prototyping machine. There is no camber to the wing, but the leading edge is rounded as a 5:1 elliptic profile. The model is mounted on a six component force and moment balance (ATI nano- 
17), which is connected to a two axis sting. The sting allows rapid pitch and plunge maneuvers to be executed, however the model was static for the measurements presented in this paper.

In previous studies ${ }^{3,4}$ the ability of pulsed-blowing jets to modify the flow around the leading edge and wing tips on low aspect ratio wings was documented. The pulsed jets acted to enhance lift by stabilizing the leading edge vortex. The same active flow control actuators placed along the leading edge of the wing are used in the current experiment to modify the lift coefficient. The actuators consist of 16 micro valves (Lee Co.) embedded in the leading edge of the wing. The actuators are supplied with compressed air through a common plenum in the center of the wing. The external air supply is pressure regulated with an i-p controller. The wing is connected to the i-p regulator with flexible tubing through the sting that supports the wing model. Although each micro valve is independently controllable, in this experiment they are opened and closed in phase with one another. The bandwidth for the micro valves is $100 \mathrm{~Hz}$, but for the results in this paper the typical open-loop

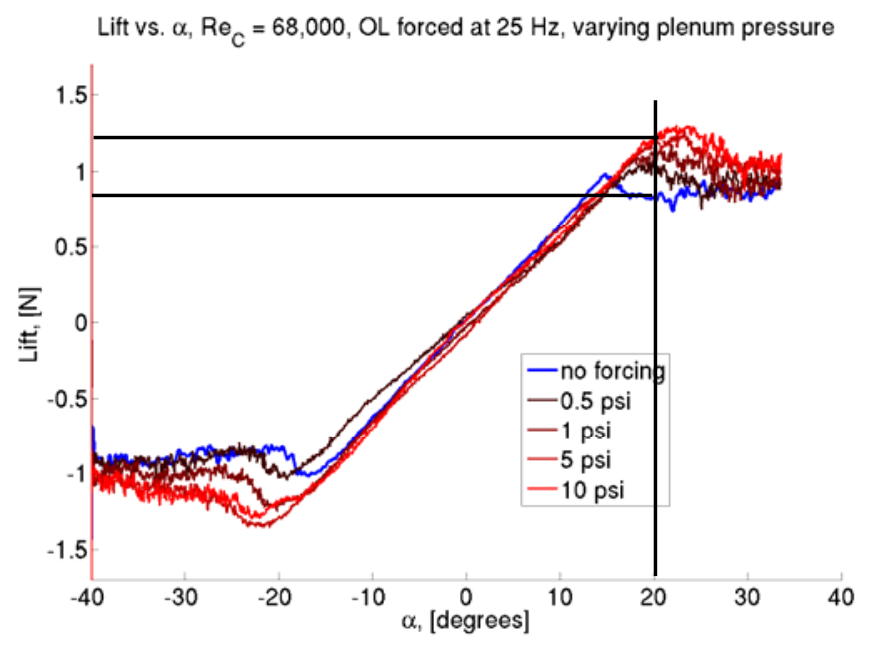

Figure 2. Lift curves for different levels of active flow control forcing amplitudes. Supply line pressures from no control (0 psi) to $10 \mathrm{psi}$ are shown. The range in lift achievable at $20^{\circ}$ angle of attack is shown by the pair of horizontal lines. operation is in the range of $29 \mathrm{~Hz}$ to $50 \mathrm{~Hz}$.

The lift dependence on angle of attack is shown in figure 2. The data was acquired by slowly pitching the wing up from $\alpha=-40^{\circ}$ to $+40^{\circ}$, at 0.5 degrees per second pitch rate. In the baseline condition without flow control the maximum lift coefficient is $\mathrm{C}_{\mathrm{Lmax}}=0.9$, and the wing stalls at $15^{\circ}$ angle of attack. With active flow control (continuous $25 \mathrm{~Hz}$ valve pulse rate), the stall angle is delayed to $\alpha=23^{\circ}$ and a higher maximum lift coefficient can be achieved, $\mathrm{C}_{\mathrm{Lmax}}=1.2$.

For the active flow control results presented in this paper, the wing was fixed at $\alpha=20^{\circ}$ which allowed a wide range of proportional control with the micro valves. The two horizontal lines superposed on the data in figure 2 show the range in lift achievable by active flow control.

Proportional control of the lift with a sufficiently large bandwidth is necessary to have a control system capable of responding to changing flight conditions. The i-p regulator controlling the supply pressure to the micro valves has a bandwidth less than $1 \mathrm{~Hz}$, which is too slow for our application. Because the micro valves are effectively in either an "on" or "off" state, they could not provide direct proportional control over the actuator jet flow

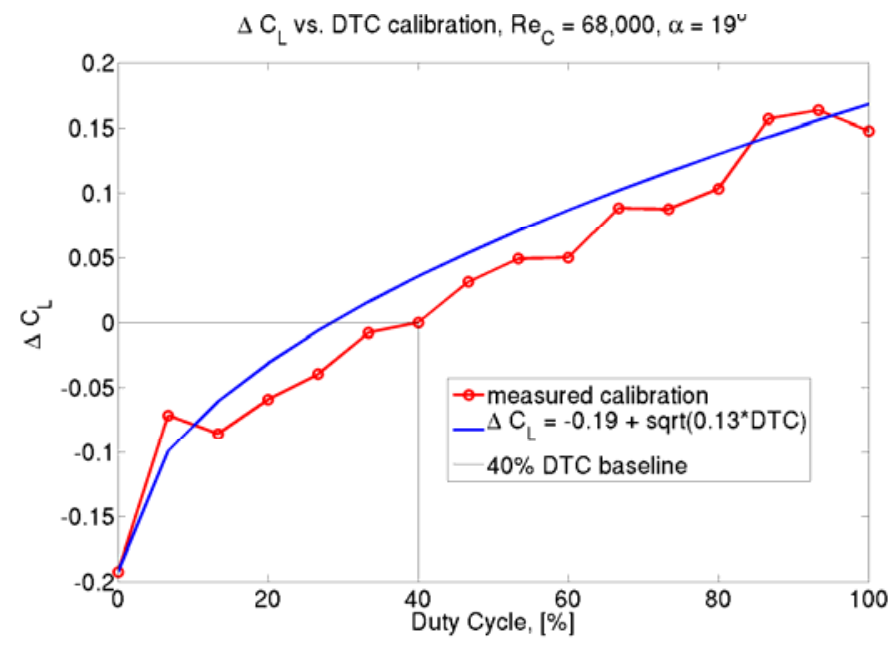

Figure 3. Lift coefficient increment dependence upon the actuator duty cycle. The nonlinear calibration must be included in the control algorithm to obtain accurate lift response to actuation.

rate. Two indirect approaches are used: (1) a

variable duty cycle (DTC) approach and (2) an amplitude modulated pulse train approach are used to achieve relatively fast proportional control of the lift.

The variable duty cycle approach, originally described in Ref. 5, is a based on step input experiments to the actuator, which showed the wing takes about $0.3 \mathrm{sec}$ to respond. With the micro valves running at $50 \mathrm{~Hz}$, then 16 different duty cycle states and lift coefficient increments can be achieved over the fundamental duty cycle period of $0.3 \mathrm{~s}$. Figure 3 shows a calibration of the increment in lift coefficient dependence on the duty cycle. A duty cycle of 
0 corresponds to the micro valves being closed over the $0.3 \mathrm{sec}$ period, while a 100 percent duty cycle indicates the valves are running continuously at $50 \mathrm{~Hz}$.

The lift increment calibration in figure 3 has a square-root dependence on the duty cycle, which must be factored into the control algorithm when producing an actuator signal. For the feed forward control experiments, the actuator was given a 40 percent bias to the duty cycle, which produces an intermediate lift increment. The bias is necessary to make it possible to create both positive and negative lift increments.

The amplitude modulated pulse train approach to modulating the lift used a $29 \mathrm{~Hz}$ square wave input as the pulse train. The amplitude was controlled by the feed forward signal from either the hot wire anemometer measurement of the freestream speed, or from the surface static pressure measured on the wing. An offset amplitude is used so that the amplitude of the pulse train varied from a "valves fully closed" to "valves fully open" state.

\section{Results}

As described in Ref. 5, the initial idea behind this set of experiments was to use a very low frequency of oscillation, so that the quasi-steady flow approximation would be valid, i.e., in the quasi-steady case,

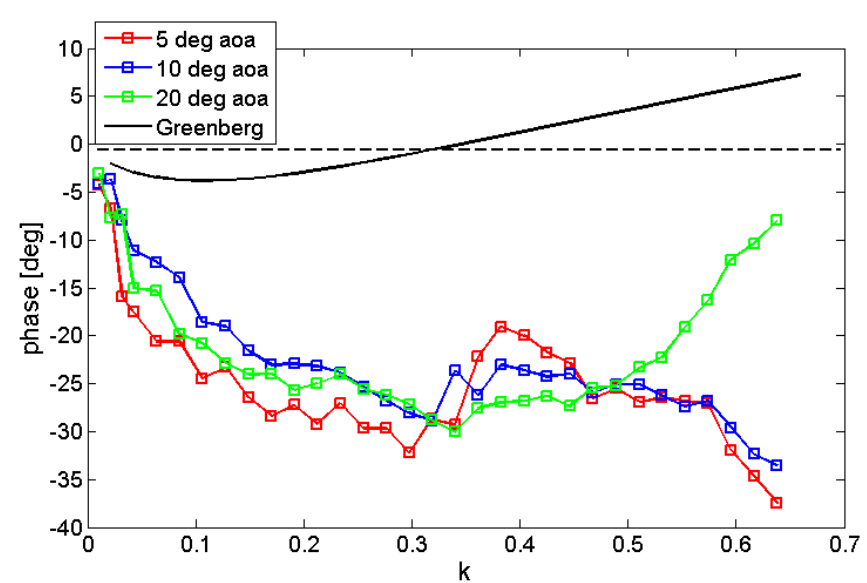

Figure 4. Phase between oscillating freestream and oscillating lift force at three different angles of attack. Prediction of Greenberg model is shown as the black line.

$L=C_{L} q S$. In particular, $\mathrm{C}_{\mathrm{L}}$ is assumed to depend only on the angle of attack and the actuator setting. With an unsteady freestream superposed on the mean flow we can write $U(t)=U_{0}+U^{\prime}(t)$. Assuming the actuation only affects the lift coefficient, then $C_{L}(t)=C_{L 0}+C_{L}^{\prime}(t)$. Substituting these expressions into the lift equation gives $L_{o}+L(t)=\frac{1}{2} \rho S\left[C_{L 0} U_{0}^{2}+\underline{2 C_{L 0} U_{0} U^{\prime}+C_{L}^{\prime} U_{0}^{2}}+\underline{\underline{C_{L 0} U^{\prime 2}+2 C_{L}^{\prime} U_{0} U^{\prime}}}+\underline{\underline{\underline{C_{L}^{\prime} U^{\prime 2}}}}\right]$

The singly underlined term is the first order component of the fluctuating lift. For harmonic oscillations of the freestream or the actuator, this term produces the fundamental peak in the lift spectrum. The double-underlined term is the second order term responsible for the first harmonic in the spectrum. The triple-underlined is the third order which is usually small. All but one of the terms can be measured directly in the experiment by turning on or off the oscillating flow in the wind tunnel or the actuators on the wing. In §III.A the freestream speed of the wind tunnel is oscillated at various frequencies, while $\mathrm{C}_{\mathrm{L}}$ ' $=0$. In $\S I I I . B$, the freestream speed is maintained constant, $U^{\prime}=0$, while the actuator is modulated to produce different frequencies of $\mathrm{C}_{\mathrm{L}}{ }^{\prime}$. The results are combined into a feed forward control algorithm for suppressing oscillations in §III.C.

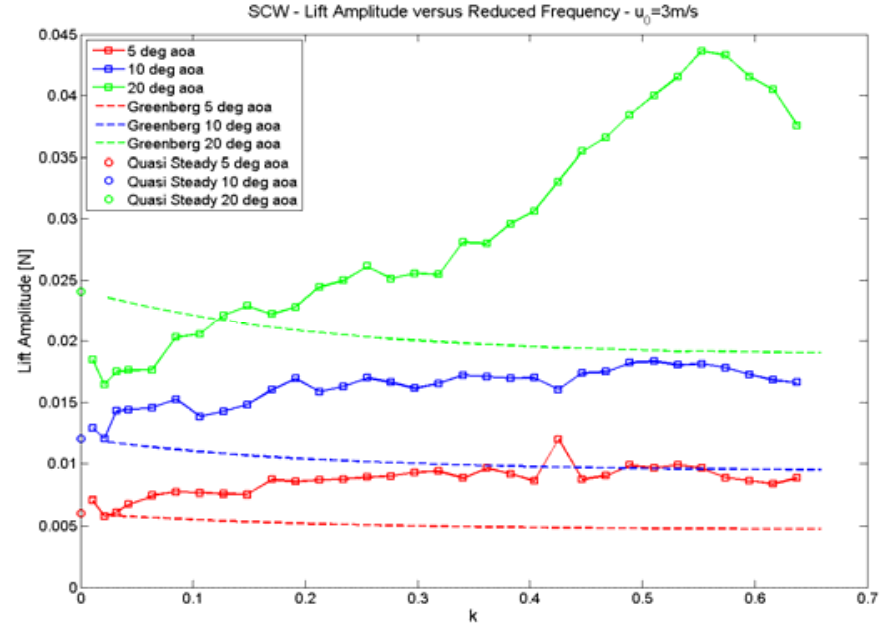

Figure 5. Peak-to-peak lift amplitude dependence on freestream speed oscillation frequency at three different angles of attack. 


\section{A. Wing Response to Unsteady Freestream Oscillation}

To document the unsteady lift behavior of the wing to the oscillating freestream, the wing was positioned at three angles of attack, $\alpha=5^{\circ}, 10^{\circ}$ and $20^{\circ}$, corresponding to fully attached flow, incipient separation and separated flow conditions, respectively. The freestream speed was oscillated about an average speed of $3 \mathrm{~m} / \mathrm{s}$ with an amplitude of $0.1 \mathrm{~m} / \mathrm{s}$, at frequencies ranging from $0.1 \mathrm{~Hz}$ to $3.0 \mathrm{~Hz}$. The freestream speed was monitored with a hot wire anemometer placed approximately 5.25 chord lengths upstream of the wing. The phase and amplitude dependence on freestream oscillation frequency were computed from the cross-spectrum of the lift signal and the freestream speed. The phase is shown in figure 4 and the peak-topeak amplitude is shown in figure 5 .

Comparisons with Greenberg's ${ }^{6}$ unsteady theory are also shown in figures 4 and 5. Greenberg's theory is an extension of Theodorsen's ${ }^{7}$ model and Isaac's model $^{8}$ for unsteady flow, which includes the effect of an oscillatory freestream. These are two-dimensional theories that account for non-circulatory (virtual mass) effects and the circulatory (wake) effects on the instantaneous lift. The theories are based on assumptions of a fully attached flow over the airfoil, a two-dimensional flow, and a planar wake. These

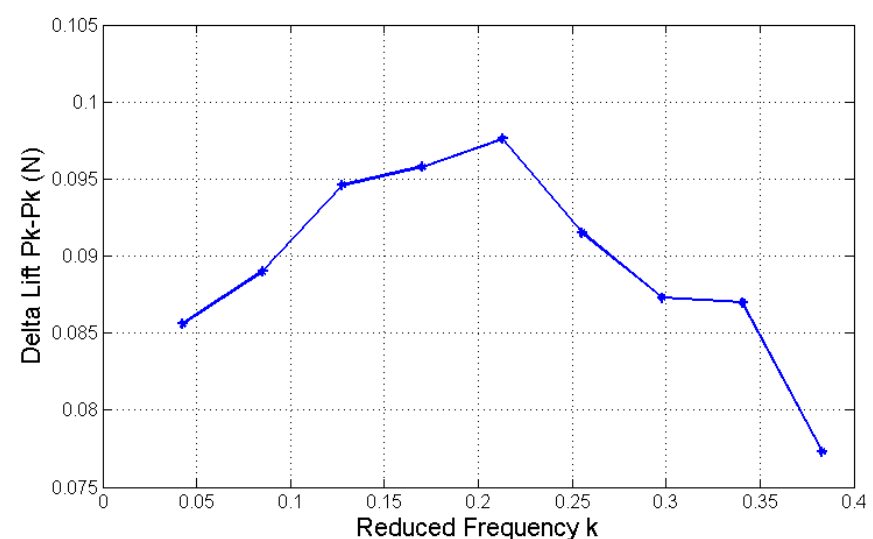

Figure 6. Peak-to-peak lift signal dependence on reduced frequency of actuation in a steady flow and fixed angle of attack $\alpha=\mathbf{2 0}^{\circ}$. assumptions are not accurate for the current experiment with the wing at $\alpha=$ $20^{\circ}$. Nevertheless, the comparison provides a useful baseline to identify the effects of three-dimensionality and flow separation on the instantaneous lift.

When the quasi-steady flow approximation is valid, then there is no phase shift between the lift and the freestream speed. However, it is clear from figure 4 that the lift lags the freestream signal by a measurable amount at even the low frequency, $0.2 \mathrm{~Hz}(\mathrm{k}=.04)$. A $20^{\circ}-30^{\circ}$ phase shift occurs over a wide range at the higher oscillation frequencies. The phase dependence does not show a strong dependence on the angle of attack, even in the separated flow case at $\alpha=20^{\circ}$.

The peak-to-peak fluctuating lift amplitude shown in figure 5 again compares the measurements at three angles of attack to the predictions of a slightly modified version of Greenberg's theory. The theory was modified to include the experimentally measured lift curve slope value of $C_{\mathrm{L}, \alpha}=2.9 / \mathrm{rad}$, instead of the ideal $\mathrm{C}_{\mathrm{L}, \alpha}=2 \pi$. This modification takes into account the finite span of the wing. The much lower value of the lift-curve slope is the result of the low aspect ratio of the wing. Ref. 9 surveyed the literature for measurements of the lift curve slope of a variety of low aspect ratio wings and made comparisons with several theoretical predictions. They found good agreement between the experiments and the equation $C_{L, \alpha}=\frac{a_{0}}{1+\left(a_{0} / \pi A R\right)(1+\tau)}$ when using $\tau=0.05$ and $\mathrm{a}_{0}=$ 5.375/rad. This equation predicts $\mathrm{C}_{\mathrm{L}, \alpha}=3.1 / \mathrm{rad}$ for the semicircular wing, which is within 4 percent of the measured value. The quasi-steady, peak-to-peak lift values are predicted using equation 1, and are plotted as circles on the ordinate in figure 6. The Greenberg model predictions for lift amplitude are shown by the dashed lines, and extrapolate to the quasi-steady values as k approaches zero.

\section{B. Wing response to Unsteady Actuation}

The time response of the wing's lift to changes in actuation is equally important as the lift response to changes in flight conditions, and must be characterized. A series of open loop forcing experiments were conducted at a constant freestream speed, $U=3.0 \mathrm{~m} / \mathrm{s}$. The lift was modulated by convolving a sine wave with the $29 \mathrm{~Hz}$ square wave pulse train driving the pulsed blowing actuators. The wing was fixed at $\alpha=20^{\circ}$, and the supply pressure to the actuators was kept constant at 4 psi. 
The peak-to-peak lift increment is shown as a function of the dimensionless frequency in figure 6. A maximum is found at $\mathrm{k}=0.2$, which corresponds to $\mathrm{f}^{+}=0.064$. Earlier work with continuously pulsed actuators showed the maximum increment in lift could be achieved at $\mathrm{f}^{+} \sim 1.0$. But it should be recognized that the results shown in figure 6 are for an amplitude modulated pulse train, which is different from the continuously pulsed (100 percent duty cycle) situation.

The phase angle between the actuator signal and the lift force is shown in figure 7. In figure 7a the phase is plotted against the actual frequency in Hertz, while in figure $7 \mathrm{~b}$ the phase is plotted against the dimensionless $\mathrm{k}$. The constant slopes for the phase data shown in figure $7 \mathrm{~b}$ indicates that the time delays are constant when normalized by the convective time scale, c/U. The linear variation of phase with frequency indicates a constant time delay. By fitting lines to the $3 \mathrm{~m} / \mathrm{s}$ and $5 \mathrm{~m} / \mathrm{s}$ data the time delays were found to be $.34 \mathrm{~s}$ and $.23 \mathrm{~s}$, respectively. Normalization with the chord and freestream speed gives $\tau^{+}=\mathrm{t}_{\text {delay }} \mathrm{U} / \mathrm{c}=5.3 \pm 0.4$.

The constant time delay response to actuation is significantly different than the response of the same wing to oscillations in the free stream. Flow visualization and surface pressure studies show that the actuator creates a train of disturbances in the separated shear layer that convect downstream at approximately half of the freestream speed. The higher freestream speeds lead to shorter delay times as shown in figure $7 \mathrm{a}$. The scaling shows that the lift requires a relatively long time to respond to the actuator input. Disturbances in the shear layer will travel approximately 2.7 chord lengths during the actuator delay time. We suspect the long time delay is at least partially associated with the viscous diffusion times of unsteady vorticity from the surface of the wing, and to some degree its convection into the wake.

\section{Feed Forward Control}

The objective of the feed forward control demonstration is to suppress the lift oscillations caused by the changes in the wind tunnel speed. Even in the quasi-steady case the lift depends on the square of the freestream speed, so the first harmonic of the freestream oscillation will play a role. The instantaneous freestream speed is measured with a hot wire anemometer, and then the desired lift coefficient to maintain a constant lift force is determined by inverting Eq. (1). The lift coefficient increment required is

$$
C_{L}^{\prime}=\frac{-2 C_{L 0} U^{\prime}}{U_{0}+2 U^{\prime}}
$$

The duty cycle required to produce the $\mathrm{C}_{\mathrm{L}}$ ' the actuator is obtained by inverting the calibration shown in figure 3. The ability of this control algorithm to suppress both fundamental and first harmonic disturbances produced by the oscillating freestream was shown by Williams, et al.
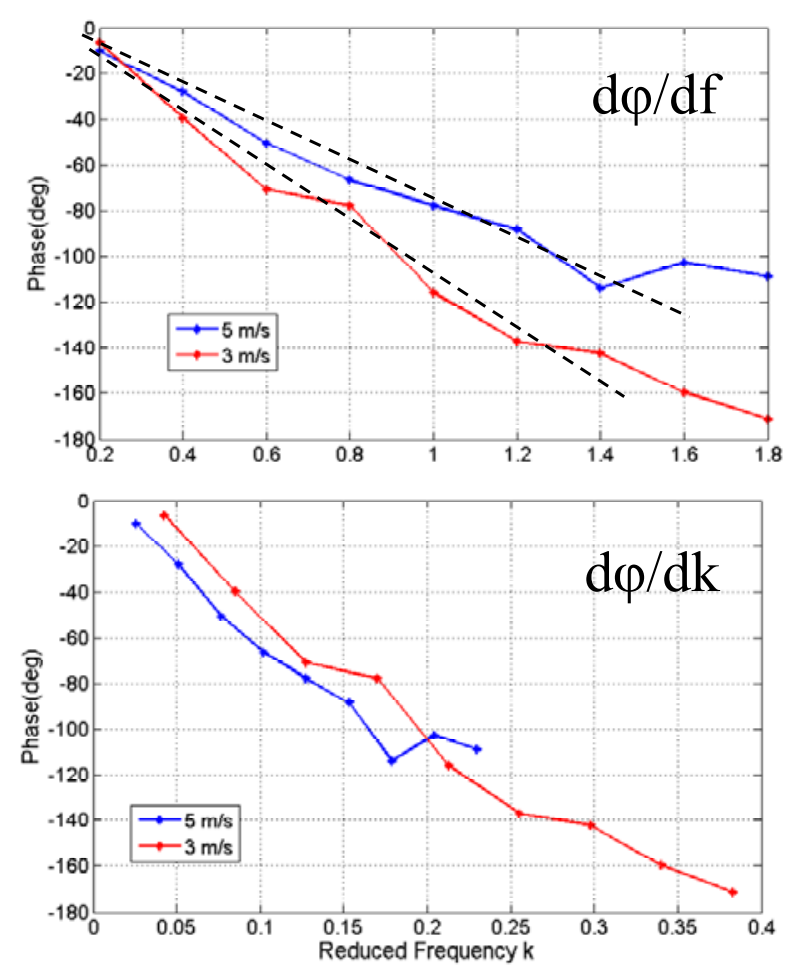

Figure 7. Phase of the lift signal at different open loop forcing frequencies. (a) phase vs. frequency (Hz); (b) phase vs. $\mathrm{k}$.

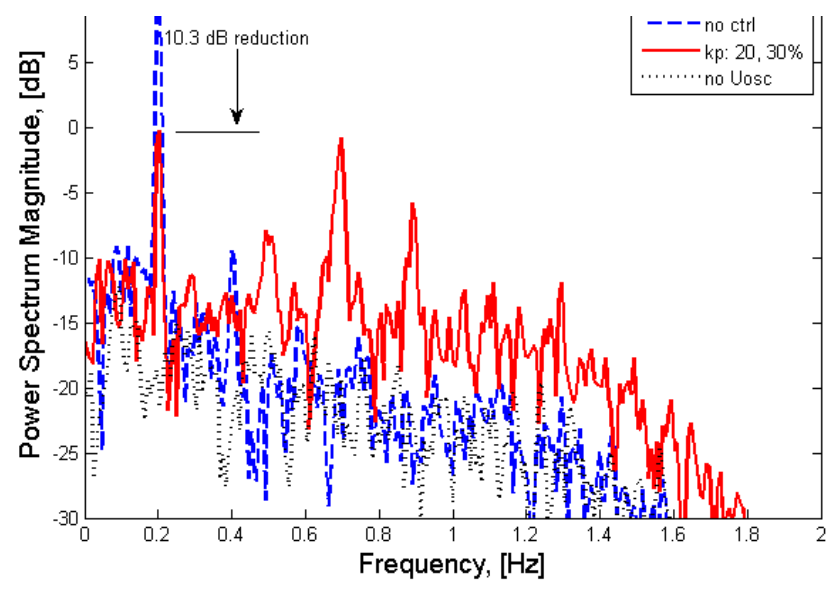

Figure 8. Spectra of the lift signal showing $10 \mathrm{~dB}$ suppression $0.2 \mathrm{~Hz}$ freestream oscillation frequency.

This approach was applied to the freestream 
oscillating at $0.2 \mathrm{~Hz}$, and the wing fixed at $\alpha=19^{\circ}$. The spectra corresponding to before and after control are shown in figure 8. The dotted-line is the baseline spectrum obtained with the wind tunnel running at a steady $5.25 \mathrm{~m} / \mathrm{s}$. The dashed-line spectrum corresponds to the wind tunnel oscillating at $0.2 \mathrm{~Hz}$, and no control is applied to the wing. Note that the lift spectrum shows peaks at the fundamental and first harmonic.

The spectrum changes to the red solid line shown in figure 6 when feed forward control is applied. The fundamental is reduced by $10 \mathrm{~dB}$, but not completely suppressed. The first harmonic is also reduced by approximately $3 \mathrm{~dB}$. However, the energy reappears as new peaks in the controlled spectrum at $0.5 \mathrm{~Hz}, 0.7 \mathrm{~Hz}$, and 0.9 Hz. The new frequencies are related to the variable duty cycle actuation scheme, and ways to suppress them is an ongoing topic of investigation.

The variable duty cycle approach is limited to lower frequencies, partially because the fundamental period of the duty cycle was $0.3 \mathrm{~s}$. Furthermore, the controller is based on the quasi-static assumption and does not account for time delays of the flow or the actuation. A different control approach was used which accounts for the time delays associated with the flow oscillation and the

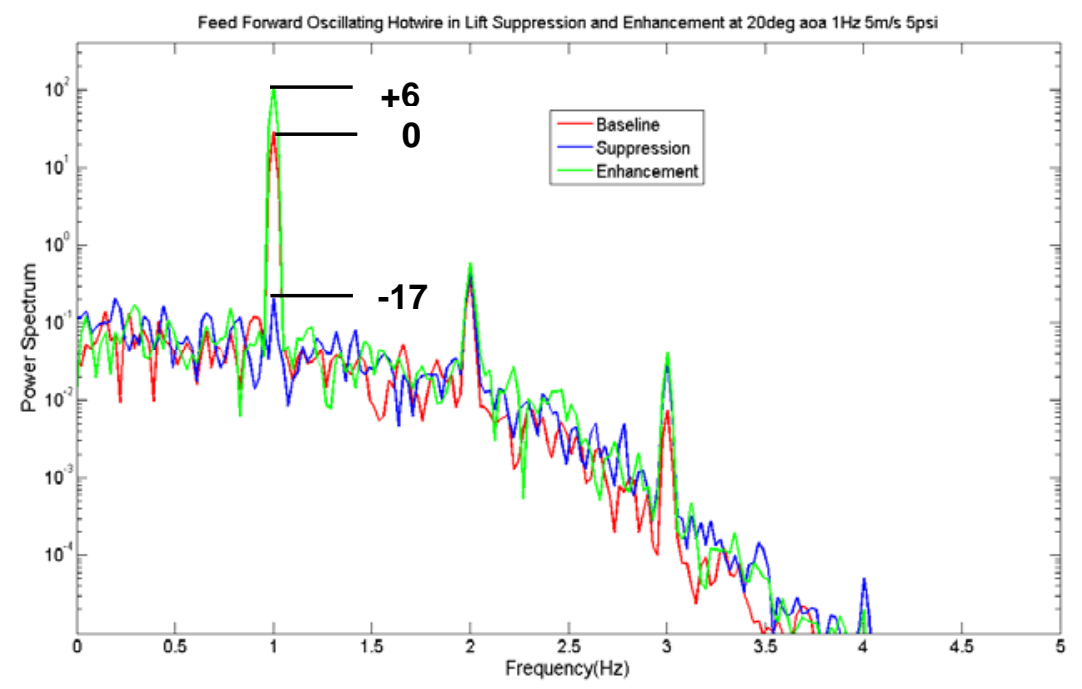

Figure 9. Feed forward control using an amplitude modulated hot wire anemometer signal with phase delay. Spectra of the lift signal showing a suppressed lift oscillation frequency (blue) and enhanced case (green) at $1 \mathrm{~Hz}$. actuation.

Instead of the variable duty cycle approach, the signal from the hot wire was used as the feed forward signal to amplitude-modulate the $29 \mathrm{~Hz}$ pulse train. Furthermore, a faster, $1 \mathrm{~Hz}$ frequency free stream oscillation was used, which was well above the ability of the variable duty cycle controller. The phase shifts measured in figures 4 and 7 were combined to produce a fluctuating lift that was $180^{\circ}$ out of phase with the lift produced by the oscillating free stream. The influence on the spectrum is shown in figure 9. The red spectrum corresponds to the uncontrolled baseline, and the blue line shows the suppressed case at $1 \mathrm{~Hz}$. This approach produced $17 \mathrm{~dB}$ of suppression at the free stream oscillation frequency of $1 \mathrm{~Hz}$, by accounting for the system lag and the actuator lag, we are able to achieve an even larger degree of suppression in the fluctuating lift than with the quasi-steady approach.

As an additional demonstration of the role of the time lags, the actuator phase was increased by $180^{\circ}$ to produce a lift enhancing signal. A $6 \mathrm{~dB}$ enhancement of the oscillating lift is seen in with the green data line in figure 9 .

The oscillation of the free stream is also detected by the surface pressure transducers. The feasibility of using the surface pressure at $\mathrm{x} / \mathrm{c}=0.4$ as a feed forward control signal is demonstrated in figure 10. Again two different time lags were selected, one corresponding to suppression of the fluctuating lift and the other producing an enhancement.

\section{Conclusions}

Experiments were conducted to investigate the feasibility of using active flow control to suppress the fluctuations in lift associated with an oscillating freestream. Two time (or phase) delays were shown to be significant factors in the problem. The phase between the lift force and the oscillating free stream was shown to be significantly longer than that predicted by classical unsteady flow theory. The time delay between the actuator and the lift response was shown to be a constant when normalized by the convective time scale. The two time delays were combined to produce a feed forward controller that suppressed the lift oscillations at the frequency of the freestream oscillations. The approach was superior to the quasi-steady model, which did not account for time lags. The use of a surface pressure signal as a replacement for the hot wire signal in the feed forward controller was demonstrated. 


\section{Acknowledgments}

The support for this work by the U.S. Air Force Office of Scientific Research MURI (FA9550-05-0369) with program manager Dr. Fariba Fahroo is gratefully appreciated. We also acknowledge the support from the Illinois NASA Space Grant Consortium for Wes Kerstens.

\section{References}

${ }^{1}$ Hoblit, F.M., Gust Loads on Aircraft: Concepts and Applications, AIAA Education Series, Washington D.C., 1988.

${ }^{2}$ Saxena, L.S., Fejer, A. A., and Morkovin, M.V., "Features of Unsteady Flows Over Airfoils," Paper No. 22, Proc. AGARD Conf. on Unsteady Aerodynamics, AGARD CP-277, 1977.

${ }^{3}$ Williams, D.R., Doshi, S., Collins, J., Colonius, T., "Control of the Spanwise Distribution of Circulation on NACA 0012 and Flat Plate Wings," AIAA Paper 2007-1121.

${ }^{4}$ Colonius, T., Rowley, C.W., Tadmor, G., Williams, D.R., Taira, K., "Closed-loop Control of leading-edge and tip vortices for small UAV," $1{ }^{\text {st }}$ Conference on Active Flow Control, Berlin Germany, Sept. 27-29, 2006.

${ }^{5}$ Williams, D.R., Collins, J., Tadmor, G., and Colonius, T., "Control of a Semi-Circular Planform Wing in a "Gusting" Unsteady Free Stream Flow: I - Experimental Issues,” AIAA Paper 2008-3976.

${ }^{6}$ Greenberg, J.M., “Airfoil in Sinusoidal Motion in a Pulsating Stream,” NACA TN-1326, 1947.

${ }^{7}$ Theodorsen, T., "General Theory of Aerodynamic Instability and the Mechanism of Flutter," NACA Rep. No. $496,1935$.

${ }^{8}$ Isaacs, R., "Airfoil Theory for Flows of Variable Velocity,” J. Aero. Sci., Vol. 12, No. 1, 1945, pp. 113-118.

${ }^{9}$ Torres, G.E., and Mueller, T.J., "Aerodynamics of Low Aspect Ratio Wings," Chapter 7 in Fixed and Flapping Wing Aerodynamics for Micro Air Vehicle Applications, Vol. 195 Prog. in Astro and Aero., 2001, pp. 115-141. 